\title{
Collection asymmetry in a drift-driven p-i-n solar cell
}

\author{
J.M. Asensi, D. Soler, M. Fonrodona, J. Bertomeu and J. Andreu \\ Departament Física Aplicada i Òptica, Universitat de Barcelona, \\ Avinguda Diagonal 647, E-08028, Barcelona, Spain
}

\begin{abstract}
An analytical expression for the voltage dependence of the internal collection efficieny of amorphous silicon p-i-n solar cells is presented. The influence of arbitrary drift lengths (asymmetrical case) is taken into account and it is shown how this asymmetry affects the spectral dependence of the collection. A new experimental techinque is proposed to determine the so-called collection voltage. Comparison between preliminary experimental data and theoretical results reveals the limitations of the analytical description.
\end{abstract}

\section{Introduction}

In the case of a-Si:H based p-i-n solar cells photocurrent depends on bias voltage $(V)$. It is because in $\mathrm{p}-\mathrm{i}-\mathrm{n}$ structures photocurrent is mainly produced in the i-layer, where the field supports photocarrier collection. The field diminishes under increasing forward voltage. This enhances the recombination and lets the photocurrent decrease.

Preprint submitted to Elsevier Science

9 July 2001 
Various analytical approaches have been proposed to describe the voltage dependence of collection in terms of the quality of the i-layer [1-3]. These models require strong assumptions in order to solve the transport equations. For instance, it is usual to assume that the electric field through the i-layer is uniform and, in many cases, only conditions of weakly absorbed light are considered. Another useful hypothesis is to assume symmetric collection [2], i.e., that the drift lengths for electrons and holes are the same. Seemingly, in the case of a-Si:H there is an experimental justification in assuming symmetric collection [4]. However, there is no difficulty about developing the more general case of arbitrary drift lengths (or $\mu \tau$-products) for electrons and holes.

In this work we review the concept of collection voltage, which is a very useful parameter to evaluate the performance of drift-driven solar cells (see Ref. [2]). We present analytical expressions for the spectral dependence of this parameter in the general case of unequal drift lenghts.

\section{The collection voltage $V_{c}$}

A straightforward experiment that demonstrates the sensitivity of photocurrent to the applied voltage is the variable illumination measurement of the short-circuit resistance $R_{s c}$, i.e., the reciprocal slope of the $I(V)$ curve at the short-circuit point. Over a wide range of illumination levels, $R_{s c}$ is inversely proportional to the short-circuit current $I_{s c}$. In other words, the $R_{s c} I_{s c}$-product is independent of the light intensity. This means that the dependence of $R_{s c}$ on light intensity is just counteracted by the dependence of $I_{s c}$ (which should be proportional to the light intensity). Thus, it follows that $R_{s c}$ must be determined by the slope of the photocurrent-voltage curve. The $R_{s c} I_{s c}$-product 
can be evaluated as

$$
R_{s c} I_{s c}=\chi\left(\frac{d \chi}{d V}\right)_{V=0}^{-1}
$$

where $\chi$ is the internal collection efficiency. The $R_{s c} I_{s c}$-product has been called collection voltage $\left(V_{c}\right)$ by Hof et al. [2]. The measurement of $V_{c}$ may be considered as an alternative to the measurement of the internal collection efficiency $\chi$. In fact, equation (1) is valid for many kinds of solar cells. The only requirement is that $R_{s c}$ must be determined by the voltage dependence of the photocurrent; i.e., not by technological factors (e.g., parallel resistance, see Ref. [1]).

In the case of a-Si:H, from analytical descriptions of the collection mechanism in $\mathrm{p}-\mathrm{i}-\mathrm{n}$ structures, plain expressions for $V_{c}$ have been deduced [1-3]. These models allow to interpret the measurement of $V_{c}$ in terms of parameters related to the quality of the material of the i-layer (e.g., $\mu \tau$-products or drift lenghts). All these approaches are inspired by the Hubin and Shah's model [5], whose main assumptions are: (A) uniform field, (B) negligible diffusion and (C) the linear approximation for the recombination function:

$$
R=\frac{p_{f}}{\tau_{p}^{0}}+\frac{n_{f}}{\tau_{n}^{0}}
$$

where $p_{f}$ and $n_{f}$ are the densities of free carriers, and $\tau_{p}^{0}$ and $\tau_{n}^{0}$ are the capture times of free carriers by neutral dangling bonds.

There have been attemps to extend this theory to more general situations: e.g., variable electric field within the i-layer [2] and charged defects near the p-i and i-n interfaces [3]. However, perhaps in order to symplify the mathematics, only very particular cases have been studied: low-recombination [3], symmetric 
collection $\left(\mu_{p} \tau_{p}^{0}=\mu_{n} \tau_{n}^{0} \equiv \mu \tau^{0}[2]\right)$ and, in all cases, uniformly absorbed light.

As commented in the Introduction, there is no special difficulty about developing the more general case. In the next Section we show the expressions for $\chi$ and $V_{c}$ in the unsymmetrical case and for non-uniformly absorbed light.

\section{Spectral dependence of $V_{c}$}

We assume an exponential carrier generation profile $G(x)$. For instance, when the cell is illuminated through the p-layer it can be expressed as follows

$$
G(x)=\phi_{o} \alpha \exp (-\alpha x)
$$

where $\alpha$ is the absorption coefficient in the i-layer and $\phi_{0}$ is the photon flux at the p-i interface $(x=0)$. As in Hubin and Shah's model, we assume that inside the i-layer bulk recombination is determined by neutral dangling bonds (eq. (2)). This may be valid at the limit of very high illumination (see [3]). Then, considering the three basic assumptions (A-C) explained earlier, the transport equations can take the following form

$$
\begin{aligned}
& L_{p} \frac{d p_{f}}{d x^{\prime}}-p_{f}\left(x^{\prime}\right)-\frac{\tau_{p}^{0}}{\tau_{n}^{0}} n_{f}\left(x^{\prime}\right)=-G\left(x^{\prime}\right) \tau_{p}^{0}, \\
& L_{n} \frac{d n_{f}}{d x^{\prime}}+\frac{\tau_{n}^{0}}{\tau_{p}^{0}} p_{f}\left(x^{\prime}\right)+n_{f}\left(x^{\prime}\right)=G\left(x^{\prime}\right) \tau_{n}^{0},
\end{aligned}
$$

where the position $x^{\prime}$ has been normalized to the i-layer thickness $\left(x^{\prime}=x / d\right)$ and, $L_{p}$ and $L_{n}$ are also normalized drift lengths: $L_{p}=\mu_{p} \tau_{p}^{0}\left(V_{b i}-V\right) / d^{2}$ and $L_{n}=\mu_{n} \tau_{n}^{0}\left(V_{b i}-V\right) / d^{2}$ ( $V_{b i}$ is the built-in voltage). The boundary condition at the interfaces p-i $\left(x^{\prime}=0\right)$ and i-n $\left(x^{\prime}=1\right)$ are defined by the effective surfacerecombination velocities. These velocities for minority carriers are assumed to 
be zero. Hence $n_{f}(0)=0$ and $p_{f}(1)=0$. We can now solve the set of equations (4) and obtain solutions for $n_{f}$ and $p_{f}$. From these solutions we are able to calculate the photocurrent and, dividing by the total generation current in the i-layer, we have the internal quantum efficiency:

$$
\chi\left(L_{a}\right)=\frac{\exp \left(1 / L_{n}\right)-\exp \left(1 / L_{a}\right) \exp \left(1 / L_{p}\right)}{\left(L_{a}-L_{c}\right)\left(\exp \left[1 / L_{a}\right]-1\right)\left(\exp \left[1 / L_{n}\right] / L_{n}-\exp \left[1 / L_{p}\right] / L_{p}\right)},
$$

where we introduce the normalized absorption length $L_{a}=\alpha d$ and the $L_{c}$ parameter (which is similar to the one defined in Ref. [5]):

$$
L_{c}=\frac{L_{p} L_{n}}{L_{p}-L_{n}}
$$

Finally, differentiating Eq. (5) with respect to the applied voltage and making use of Eq. (1) we obtain $V_{c}$ :

$$
V_{c}\left(L_{a}\right)=\frac{L_{c} V_{b i}}{\left(1-\frac{L_{n}}{L_{p}} \exp \left[-1 / L_{c}\right]\right)^{-1}-\frac{L_{c} L_{a}}{L_{c}-L_{a}}+\left(\exp \left[1 / L_{a}-1 / L_{c}\right]-1\right)^{-1}} \cdot(7)
$$

From (5) and (7) we can study some particular situations: e.g., the uniform illumination case can be obtained when $L_{a}$ becomes infinite. Table 1 summarizes some results for these particular situations.

We may note that, when we study from (5) and (7) the spectral dependence of $\chi$ and $V_{c}$, there are three different behaviours that can be identified according to the relation between $L_{p}$ and $L_{n}$ (see Fig.1):

- If $L_{p}=L_{n}$ (symmetric case) then $\chi$ and $V_{c}$ are not wavelength dependent.

- If $L_{p}<L_{n}\left(L_{c}<0\right)$ then $\chi$ and $V_{c}$ decrease monotonously with growing wavelengths (when $L_{a}$ increases).

- If $L_{p}>L_{n}\left(L_{c}>0\right)$, then $\chi$ and $V_{c}$ increase monotonously with growing 
wavelengths.

\section{New experimental procedure and results}

As we have seen in Secc. 2, the value of $V_{c}$ is determined by measuring $I_{s c}$ and $R_{s c}$. Several methods have been proposed to evaluate $R_{s c}$ : e.g., best linear fit of $I(V)$ at $V=0$ [1] or ac variable-illumination-measurement [2]. However the use of these methods is limited when $R_{s c}$ is affected by parasitic effects. For instance, at high illumination levels the $R_{s c}$ value may be related to the parallel resistance and not to the voltage-dependent photocurrent collection. In this work, we propose a procedure based on measuring directly the photocurrent change when a bias voltage is applied to the device.

We begin with a typical spectral response measurement in which a probelight of wavelength $\lambda$ is chopped and the resulting ac current $I_{\lambda}$ from the cell is detected by lock-in technique. A photocurrent change $d I_{\lambda}$ can be detected when a small voltage $d v$ is applied. Then, according to eq. (1), $V_{c}$ may be calculated by $I_{\lambda} d v / d I_{\lambda}$. We can, thus, obtain the spectrally resolved value of $V_{c}$ for a given bias-light condition. However, the measurement of the change $d I_{\lambda}$ may be not very precise in the case of good cells with high values of $V_{c}$. In order to accurately determine $d I_{\lambda}$ we propose (as in Ref. [2]) to modulate the applied voltage. The method of measurement is illustrated in Fig. 2. Two alternating signals at different frequencies are applied to the test cell: a monochromatic beam at frequency $f_{1}$ and the ac voltage $d v$ at frequency $f_{2}$. With a first lockin amplifier (LIA1) whose reference is taken from the light chopper $\left(f_{1}\right)$ we detect the photocurrent $I_{1}$ (or $V_{1}$ in Fig. 2). If $f_{1}$ is sufficiently higher than $f_{2}$ then, the output from LIA1 can be smoothed by a suitable filter. Thus, this 
output is proportional to the alternating photocurrent due to the ac voltage. Measuring the LIA1 output with a second lock-in (LIA2) whose reference is $f_{2}$ we can detect the photocurrent change. This method allows an accurate determination of $V_{c}$ as a function of wavelength and at different bias-light intensities.

Figure 3 shows how the $V_{c}$ measurement for two typical a-Si:H p-i-n solar cells (cells A and B) varies with wavelength. There are several important points to note (from these very preliminary results):

- $V_{c}$ is wavelength-dependent.

- This dependence is not monotonous (we find a maximum when the normalized absorption length is about 1).

- $V_{c}$ is sensitive to the bias-light intensity.

\section{Discussion and concluding remarks}

By performing a new experimental technique we have obtained preliminary results about the spectral dependence of $V_{c}$. These results show that the light wavelength does affect the $V_{c}$ value. This seems not to be compatible with a symmetric collection model. The asymmetric description (arbitrary drift lenghts for electron and holes), in which most of the dangling bonds are in the neutral state, also shows discrepancies: the monotonous wavelengthdependence is not observed in the experimental data.

The analytical model should be refined taking into account some new effects. For instance, it is probable that the charged regions of the i-layer should be taken into consideration. We should note that this assumption, as discussed 
in our previous work (see Ref. [3]), could explain the observed sensitivity of $V_{c}$ to the bias-ligth.

\section{References}

[1] J. Merten, J.M. Asensi, C. Voz, A.V. Shah, R. Platz and J. Andreu. IEEE Trans. Electron. Dev. 45 (2) (1998) 423.

[2] Ch. Hof, N. Wyrsch and A.V. Shah. J. Non-Cryst. Solids 266-269 (2000) 1114.

[3] J.M. Asensi, J. Merten, C. Voz and J. Andreu. J. Appl. Phys. 85 (5) (1999) 2939.

[4] N. Beck, N. Wyrsch, Ch. Hof and A.V. Shah J. Appl. Phys. 79 (12) (1996) 9361.

[5] J. Hubin and A.V. Shah Philos. Mag. B 72 (6) (1995) 589. 


\section{Figure Captions}

- Table 1: Specific expressions for $\chi$ and $V_{c}$ deduced from eq. 5 and 7 in some particular cases.

- Figure 1: Internal quantum efficiency $\chi$ and collection voltage $V_{c}$ as a function of the normalized absorption length $L_{a}$ for varying values of the normalized drift lengths. Note that illumination through the n-layer is also considered.

- Figure 2: Schematic set-up for the measurement of the spectral dependence of the colecction voltage $V_{c}$.

- Figure 3: Collection voltage measurements for two typical a-Si:H p-i-n solar cells: cell A was deposited at higher temperature. Dashed lines are experimental results under bias-light condition (solid lines are in the dark). For cell A the measurement after light-soaking is shown ( $\left.\mathrm{A}^{\prime}\right)$. 
Table 1

$$
\begin{aligned}
& \text { General Case }\left(L_{p} \neq L_{n}\right) \text { : } \\
& \chi(\infty)=\frac{\exp \left(1 / L_{n}\right)-\exp \left(1 / L_{p}\right)}{\exp \left(1 / L_{n}\right) / L_{n}-\exp \left(1 / L_{p}\right) / L_{p}} \quad \chi(0)=\frac{\chi(\infty)}{L_{c}\left(\exp \left[1 / L_{c}\right]-1\right)} \\
& V_{c}(\infty)=\frac{V_{b i}}{1-\chi(\infty) /\left\{2 L_{c}^{2}\left(\cosh \left[\frac{1}{L_{c}}\right]-1\right)\right\}} \quad V_{c}(0)=L_{c} V_{b i}\left(1-\frac{L_{n}}{L_{p}} \exp \left[-1 / L_{c}\right]\right)
\end{aligned}
$$

Symmetric Case $\left(L_{n}=L_{p} \equiv L^{0}\right)$ :

$$
\chi\left(L_{a}\right)=\frac{L^{0}}{1+L^{0}}=c t e \quad V_{c}\left(L_{a}\right)=V_{b i}\left(1+L^{0}\right)=c t e
$$

Low-Recombination Case $(\chi \approx 1)$ :

$$
V_{c}\left(L_{a}\right)=\frac{L_{c} V_{b i}}{L_{c} / L_{n}-L_{a}+\left(\exp \left[1 / L_{a}\right]-1\right)^{-1}}
$$

$$
V_{c}(\infty)=2 L_{n} L_{p} V_{b i} /\left(L_{n}+L_{p}\right) \quad V_{c}(0)=V_{b i} L_{n}
$$

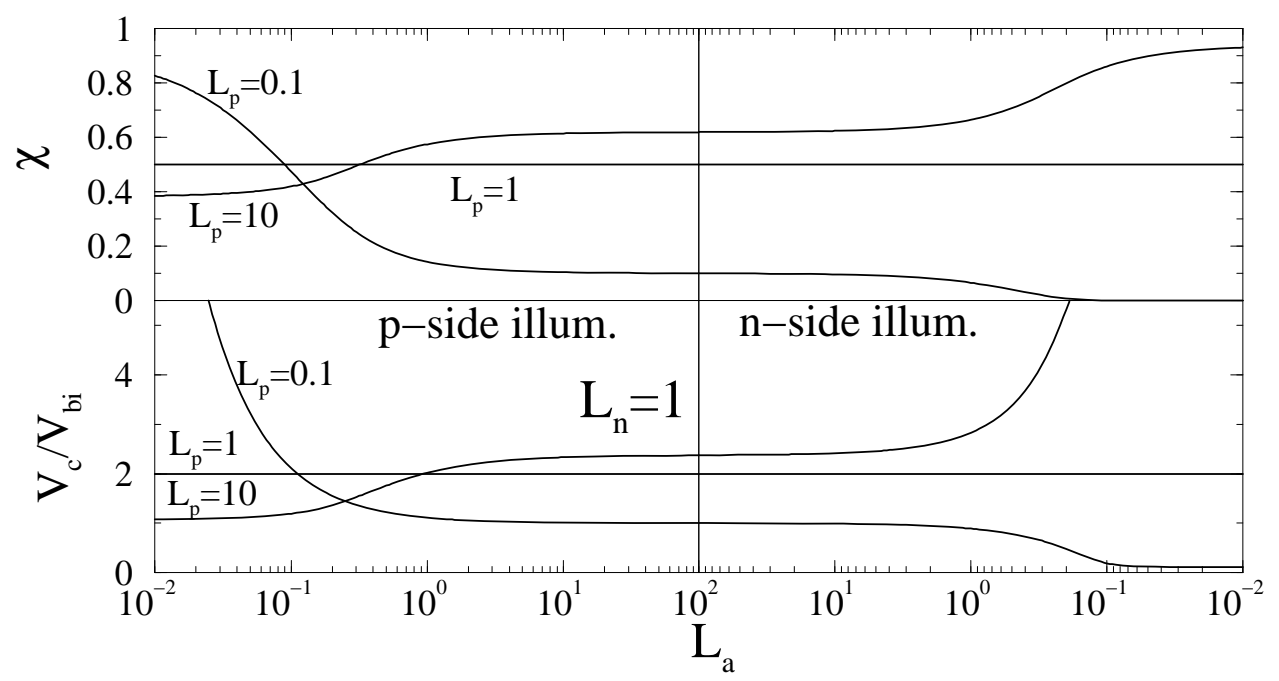

Fig. 1. 


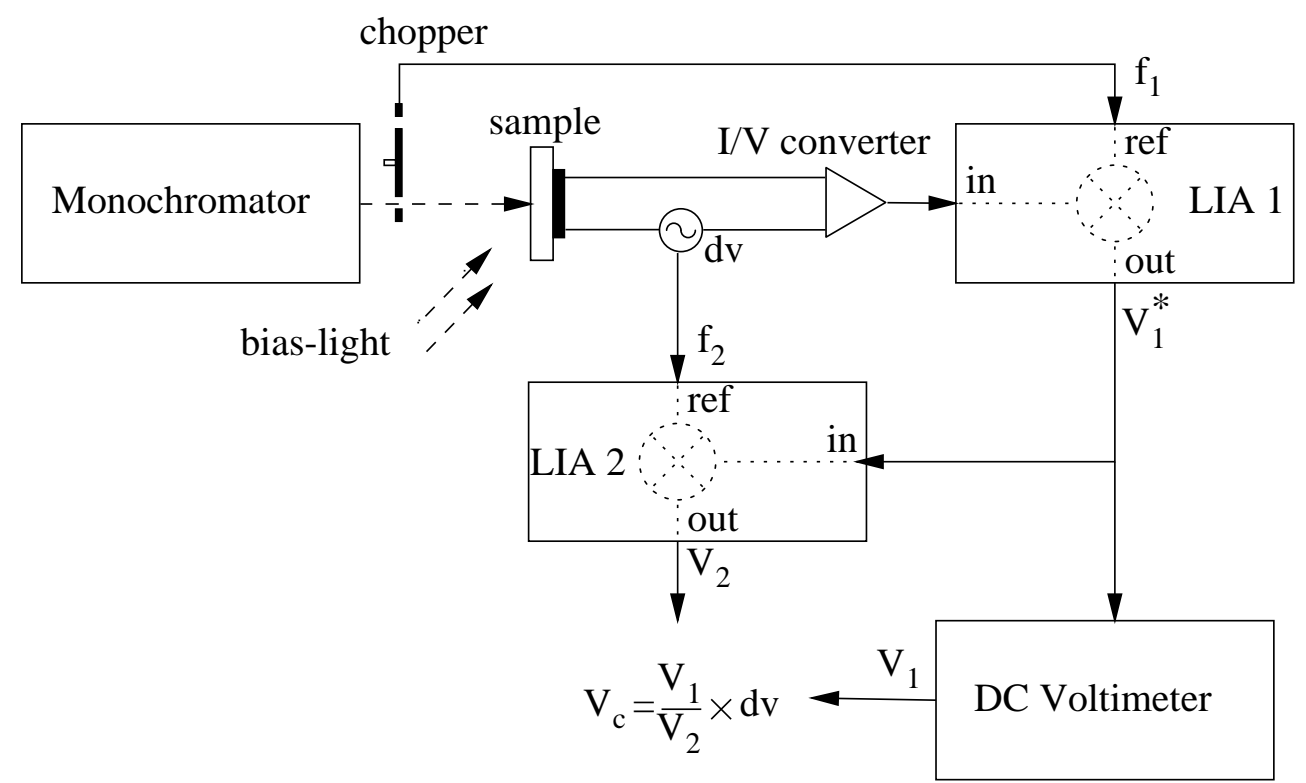

Fig. 2.

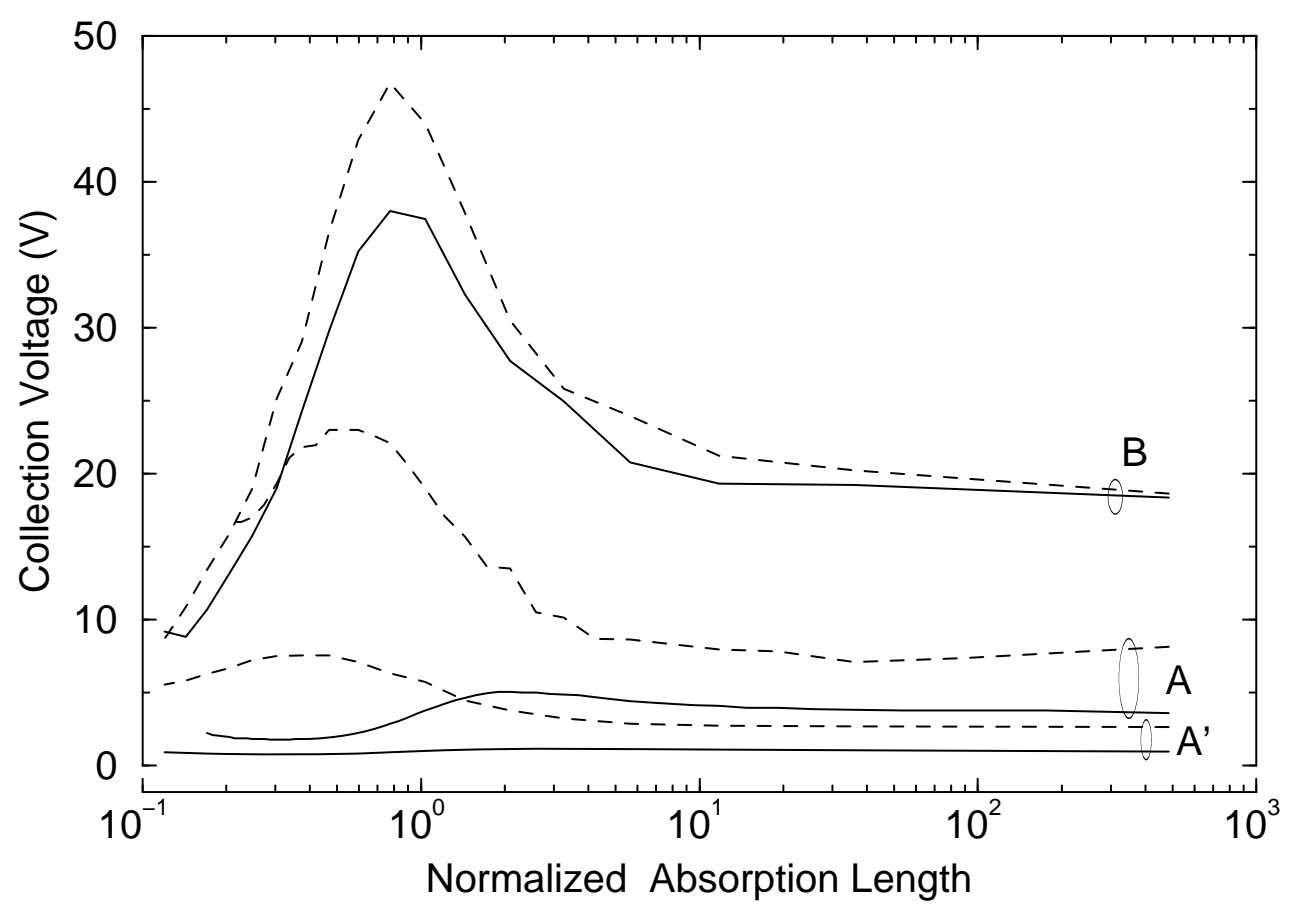

Fig. 3. 